\title{
On Green's function for boundary value problem with eigenvalue dependent quadratic boundary condition
}

Haskız Coşkun*, Ayşe Kabataş and Elif Başkaya

$\bar{~} \overline{\text { COrrespondence: haskiz@ktu.edu.tr }}$ Faculty of Science, Department of Mathematics, Karadeniz Technical University, Trabzon, 61080, Turkey

\begin{abstract}
In this paper we obtain Green's function for a regular Sturm-Liouville problem having the eigenparameter in all boundary conditions in which the left one is in quadratic form. We assume no smoothness condition on the potential.
\end{abstract}

Keywords: Sturm-Liouville problem; eigenfunction asymptotics; Green's function

\section{Introduction}

In this paper, we consider the boundary value problem

$$
\begin{aligned}
& -y^{\prime \prime}(t)+[q(t)-\lambda] y(t)=0, \\
& y^{\prime}(a)=\left(c \lambda^{2}+d \lambda+e\right) y(a), \\
& b_{1} y(b)+b_{2} y^{\prime}(b)=\lambda\left[b_{1}^{\prime} y(b)+b_{2}^{\prime} y^{\prime}(b)\right],
\end{aligned}
$$

where $\lambda$ is real parameter; $q(t)$ is a real-valued function and $q(t) \in L^{1}[a, b] ; a, b, b_{1}, b_{2}, b_{1}^{\prime}, b_{2}^{\prime}$, $c, d, e \in \mathbb{R}$ and $c \neq 0$. This problem is different from the usual regular Sturm-Liouville problem [1] in the sense that the eigenvalue parameter $\lambda$ appears in both boundary conditions and in the first boundary condition is in quadratic form. We first derive asymptotic approximations for the eigenfunctions of the problem, and then using these approximations we obtain Green's function.

One application of the Green's function is to derive sampling theorems associated with eigenvalue problems containing an eigenvalue parameter in the boundary condition [2].

We suppose without loss of generality that $q$ has a mean value zero, i.e.,

$$
\int_{a}^{b} q(t) d t=0
$$

As an illustration of our results we obtain for $a \leq \varkappa \leq \xi \leq b, b_{2}^{\prime} \neq 0$,

$$
\begin{aligned}
G(\varkappa, \xi, \lambda)= & -\lambda^{-1 / 2} \frac{\sin \lambda^{1 / 2}(\varkappa-a) \cos \lambda^{1 / 2}(b-\xi)}{\cos \lambda^{1 / 2}(b-a)}+\frac{\lambda^{-1}}{\cos \lambda^{1 / 2}(b-a)} \\
& \times\left\{\frac{1}{2}\left(\int_{a}^{\varkappa} q(t) d t\right) \cos \lambda^{1 / 2}(\varkappa-a) \cos \lambda^{1 / 2}(b-\xi)\right.
\end{aligned}
$$

(c) The Author(s) 2017. This article is distributed under the terms of the Creative Commons Attribution 4.0 International License (http://creativecommons.org/licenses/by/4.0/), which permits unrestricted use, distribution, and reproduction in any medium, provided you give appropriate credit to the original author(s) and the source, provide a link to the Creative Commons license, and indicate if changes were made. 


$$
\begin{aligned}
& -\left[\frac{b_{1}^{\prime}}{b_{2}^{\prime}}+\frac{1}{2} \int_{\xi}^{b} q(t) d t\right] \sin \lambda^{1 / 2}(\varkappa-a) \sin \lambda^{1 / 2}(b-\xi) \\
& \left.+\frac{b_{1}^{\prime}}{b_{2}^{\prime}} \tan \lambda^{1 / 2}(b-a) \sin \lambda^{1 / 2}(\varkappa-a) \cos \lambda^{1 / 2}(b-\xi)\right\} \\
& +O\left(\lambda^{-1} \eta(\lambda)\right)
\end{aligned}
$$

where $\eta(\lambda) \rightarrow 0$ as $\lambda \rightarrow \infty$. Similar results hold for $a \leq \xi \leq \varkappa \leq b$ exchanging the role of $\varkappa$ and $\xi$.

These types of results with different boundary conditions were obtained before in the case where $q(t)$ is assumed to be continuous on a finite interval [2, 3]. In [3], the Green's functions were derived asymptotically with the error term of exponential type, that is, for $\lambda=s^{2}, G(x, y, \lambda)=\cdots+O\left(\frac{1}{|s|^{2}} \exp [t(y-x)]\right), a \leq y \leq x$ (p.304). In our results, we only assume that $q(t) \in L^{1}[a, b]$ and find that $G(x, y, \lambda)=\cdots+O\left(\lambda^{-1} \eta(\lambda)\right)$ where $\eta(\lambda) \rightarrow 0$ as $\lambda \rightarrow \infty$. We use a similar approach as in [4] in which the spectral functions associated with Sturm-Liouville problems were considered on $[0, \infty)$ with usual boundary condition not including the eigenparameter for $q(t) \in L^{1}[0, \infty)$.

\section{Method}

We associate with (1.1) the Riccati equation

$$
v^{\prime}=-\lambda+q-v^{2} .
$$

It was shown in [4] that if $v(t, \lambda)$ is a complex-valued solution of $(2.1)$ and

$$
\begin{aligned}
& S(t, \lambda):=\operatorname{Re}\{v(t, \lambda)\}, \\
& T(t, \lambda):=\operatorname{Im}\{v(t, \lambda)\},
\end{aligned}
$$

then any nontrivial real-valued solution of (1.1) can be expressed as

$$
z(t, \lambda)=c_{1} \exp \left(\int_{a}^{t} S(x, \lambda) d x\right) \cos \left\{c_{2}+\int_{a}^{t} T(x, \lambda) d x\right\}
$$

with

$$
\begin{aligned}
z^{\prime}(t, \lambda)= & c_{1} S(t, \lambda) \exp \left(\int_{a}^{t} S(x, \lambda) d x\right) \cos \left\{c_{2}+\int_{a}^{t} T(x, \lambda) d x\right\} \\
& -c_{1} \exp \left(\int_{a}^{t} S(x, \lambda) d x\right) \sin \left\{c_{2}+\int_{a}^{t} T(x, \lambda) d x\right\} T(t, \lambda) .
\end{aligned}
$$

Here, $c_{1}$ and $c_{2}$ are constants to be determined.

We suppose that for each $t \in[a, b]$ there exist

$$
\left|\int_{t}^{b} e^{2 i \lambda \lambda^{1 / 2} x} q(x) d x\right| \leq A(t) \eta(\lambda)
$$

where

(i) $A(t):=\int_{t}^{b}|q(x)| d x$ is clearly a decreasing function and $A(t) \in L^{1}[a, b]$,

(ii) $\eta(\lambda) \rightarrow 0$ as $\lambda \rightarrow \infty$. 
For $q(t) \in L^{1}[a, b]$ the existence of the $A$ and $\eta$ functions may be established, for $\lambda$ positive as follows.

We note that, avoiding the trivial case $\int_{t}^{b}|q(x)| d x=0$,

$$
\left|\int_{t}^{b} e^{2 i \lambda^{1 / 2} x} q(x) d x\right| \leq \int_{t}^{b}|q(x)| d x<\infty
$$

So, if we define

$$
F(t, \lambda):= \begin{cases}\left|\int_{t}^{b} e^{2 i \lambda \lambda^{1 / 2}} q(x) d x\right| / \int_{t}^{b}|q(x)| d x, & \text { if } \int_{t}^{b}|q(x)| d x \neq 0 \\ 0, & \text { if } \int_{t}^{b}|q(x)| d x=0\end{cases}
$$

then $0 \leq F(t, \lambda) \leq 1$, and we set $\eta(\lambda):=\sup _{a \leq t \leq b} F(t, \lambda)$. Here $\eta(\lambda)$ is well defined by (2.7) and goes to zero as $\lambda \rightarrow \infty$ [4].

Our method of approximating a solution of (2.1) is similar to that of [4]. We consider (2.1) on $[a, b]$ and set

$$
v(t, \lambda):=i \lambda^{1 / 2}+\sum_{n=1}^{\infty} v_{n}(t, \lambda)
$$

Substitution of (2.8) into (2.1) and rearrangement gives

$$
\begin{aligned}
v_{1}^{\prime} & +2 i \lambda^{1 / 2} v_{1}+v_{2}^{\prime}+2 i \lambda^{1 / 2} v_{2}+\sum_{n=3}^{\infty}\left(v_{n}^{\prime}+2 i \lambda^{1 / 2} v_{n}\right) \\
& =q-v_{1}^{2}-\sum_{n=3}^{\infty}\left(v_{n-1}^{2}+2 v_{n-1} \sum_{m=1}^{n-2} v_{m}\right) .
\end{aligned}
$$

We choose the $v_{n}$ so that

$$
\begin{aligned}
& v_{1}^{\prime}+2 i \lambda^{1 / 2} v_{1}=q, \\
& v_{2}^{\prime}+2 i \lambda^{1 / 2} v_{2}=-v_{1}^{2},
\end{aligned}
$$

and for $n=3,4, \ldots$,

$$
v_{n}^{\prime}+2 i \lambda^{1 / 2} v_{n}=-\left(v_{n-1}^{2}+2 v_{n-1} \sum_{m=1}^{n-2} v_{m}\right) .
$$

Solving for $v_{n}, n=1,2,3, \ldots$,

$$
\begin{aligned}
& v_{1}(t, \lambda)=-e^{-2 i \lambda^{1 / 2} t} \int_{t}^{b} e^{2 i \lambda^{1 / 2} x} q(x) d x, \\
& v_{2}(t, \lambda)=e^{-2 i \lambda^{1 / 2} t} \int_{t}^{b} e^{2 i \lambda^{1 / 2} x} v_{1}^{2}(x, \lambda) d x, \\
& v_{n}(t, \lambda)=e^{-2 i \lambda^{1 / 2} t} \int_{t}^{b} e^{2 i \lambda^{1 / 2} x}\left(v_{n-1}^{2}+2 v_{n-1} \sum_{m=1}^{n-2} v_{m}\right) d x .
\end{aligned}
$$


It is shown in [5] that $\sum_{n=1}^{\infty} v_{n}(t, \lambda)$ is uniformly absolutely convergent for all $\lambda \geq \lambda_{0}$ and for all $t \in[a, b]$. It also follows from the choice of $v_{n}, n=1,2,3, \ldots$, that $\sum_{n=1}^{\infty} v_{n}^{\prime}(t, \lambda)$ is uniformly absolutely convergent. The series $i \lambda^{1 / 2}+\sum_{n=1}^{\infty} v_{n}(t, \lambda)$ is therefore a solution of (2.1) and

$$
\begin{aligned}
& S(t, \lambda)=\operatorname{Re} \sum_{n=1}^{\infty} v_{n}(t, \lambda), \\
& T(t, \lambda)=\lambda^{1 / 2}+\operatorname{Im} \sum_{n=1}^{\infty} v_{n}(t, \lambda) .
\end{aligned}
$$

The asymptotic forms of $S(t, \lambda)$ and $T(t, \lambda)$ are obtained in [6] as follows:

$$
S(t, \lambda)=-\sin \left(2 \lambda^{1 / 2} t+\zeta_{t}\right)+O\left(\eta^{2}(\lambda)\right)
$$

and

$$
T(t, \lambda)=\lambda^{1 / 2}-\cos \left(2 \lambda^{1 / 2} t+\zeta_{t}\right)+O\left(\eta^{2}(\lambda)\right)
$$

where

$$
\sin \zeta_{t}=\int_{t}^{b} q(x) \cos 2 \lambda^{1 / 2} x d x, \quad \cos \zeta_{t}=\int_{t}^{b} q(x) \sin 2 \lambda^{1 / 2} x d x
$$

Also,

$$
\begin{aligned}
\int_{a}^{t} S(x, \lambda) d x= & \frac{1}{2 \lambda^{1 / 2}}\left\{\cos \left(2 \lambda^{1 / 2} t+\zeta_{t}\right)-\cos \left(2 \lambda^{1 / 2} a+\zeta_{a}\right)\right\} \\
& +O\left(\lambda^{-1 / 2} \eta^{2}(\lambda)\right), \\
\int_{a}^{t} T(x, \lambda) d x= & \lambda^{1 / 2}(t-a)-\frac{1}{2 \lambda^{1 / 2}}\left\{\sin \left(2 \lambda^{1 / 2} t+\zeta_{t}\right)-\sin \left(2 \lambda^{1 / 2} a+\zeta_{a}\right)+\int_{a}^{t} q(x) d x\right\} \\
& +O\left(\lambda^{-1 / 2} \eta^{2}(\lambda)\right) .
\end{aligned}
$$

The last two equalities are obtained in [7].

\section{Approximations for the eigenfunctions}

In this section we obtain approximations for the solution of (1.1)-(1.3). We define two solutions, $\Psi(t, \lambda)$ and $\Phi(t, \lambda)$ of (1.1)-(1.3) with the initial conditions

$$
\Psi(a, \lambda)=1, \quad \Psi^{\prime}(a, \lambda)=c \lambda^{2}+d \lambda+e,
$$

and

$$
\Phi(b, \lambda)=b_{2}-b_{2}^{\prime} \lambda, \quad \Phi^{\prime}(b, \lambda)=b_{1}^{\prime} \lambda-b_{1}
$$


Theorem 1 Let $\Psi(t, \lambda)$ and $\Phi(t, \lambda)$ be the solutions of (1.1) satisfying (3.1) and (3.2), respectively. Then we find

$$
\begin{aligned}
\Psi(t, \lambda)= & \frac{1}{\cos \left\{\cot ^{-1}\left[\frac{T(a, \lambda)}{-c \lambda^{2}-d \lambda-e+S(a, \lambda)}\right]\right\}} \exp \left(\int_{a}^{t} S(x, \lambda) d x\right) \\
& \times \cos \left\{\cot ^{-1}\left[\frac{T(a, \lambda)}{-c \lambda^{2}-d \lambda-e+S(a, \lambda)}\right]+\int_{a}^{t} T(x, \lambda) d x\right\}
\end{aligned}
$$

and

(i) if $b_{2}^{\prime} \neq 0$,

$$
\Phi(t, \lambda)=\frac{\left(b_{2}-b_{2}^{\prime} \lambda\right) \exp \left(-\int_{t}^{b} S(x, \lambda) d x\right)}{\cos \left\{\tan ^{-1} F_{1}(b, \lambda)\right\}} \cos \left\{\tan ^{-1} F_{1}(b, \lambda)-\int_{t}^{b} T(x, \lambda) d x\right\}
$$

where

$$
F_{1}(b, \lambda):=\frac{1}{T(b, \lambda)}\left[S(b, \lambda)+\frac{b_{1}^{\prime} \lambda-b_{1}}{b_{2}^{\prime} \lambda-b_{2}}\right]
$$

(ii) if $b_{2}^{\prime}=0$,

$$
\Phi(t, \lambda)=\frac{b_{2} \exp \left(-\int_{t}^{b} S(x, \lambda) d x\right)}{\cos \left\{\cot ^{-1} F_{2}(b, \lambda)\right\}} \cos \left\{\cot ^{-1} F_{2}(b, \lambda)-\int_{t}^{b} T(x, \lambda) d x\right\},
$$

where

$$
F_{2}(b, \lambda):=\frac{b_{2} T(b, \lambda)}{-b_{1}^{\prime} \lambda+b_{1}+b_{2} S(b, \lambda)} .
$$

Proof of Theorem 1 From (2.4), (2.5) and (3.1), we have

$$
\begin{aligned}
& \Psi(a, \lambda)=c_{1} \cos c_{2}=1, \\
& \Psi^{\prime}(a, \lambda)=c_{1} S(a, \lambda) \cos c_{2}-c_{1} \sin c_{2} T(a, \lambda)=c \lambda^{2}+d \lambda+e .
\end{aligned}
$$

From (3.3), we obtain

$$
c_{1}=\frac{1}{\cos c_{2}} \text {. }
$$

Using this equation in (3.4),

$$
c_{2}=\cot ^{-1}\left[\frac{T(a, \lambda)}{-c \lambda^{2}-d \lambda-e+S(a, \lambda)}\right] .
$$

Hence

$$
c_{1}=\frac{1}{\cos \left\{\cot ^{-1}\left[\frac{T(a, \lambda)}{-c \lambda^{2}-d \lambda-e+S(a, \lambda)}\right]\right\}} .
$$

Substituting the values of $c_{1}$ and $c_{2}$ into (2.4), we evaluate $\Psi(t, \lambda)$ as required. In order to find $\Phi(t, \lambda)$, 
(i) if $b_{2}^{\prime} \neq 0$, we obtain from (2.4), (2.5) and (3.2)

$$
\begin{aligned}
\Phi(b, \lambda)= & c_{1} \exp \left(\int_{a}^{b} S(x, \lambda) d x\right) \cos \left\{c_{2}+\int_{a}^{b} T(x, \lambda) d x\right\} \\
= & b_{2}-b_{2}^{\prime} \lambda, \\
\Phi^{\prime}(b, \lambda)= & c_{1} S(b, \lambda) \exp \left(\int_{a}^{b} S(x, \lambda) d x\right) \cos \left\{c_{2}+\int_{a}^{b} T(x, \lambda) d x\right\} \\
& -c_{1} \exp \left(\int_{a}^{b} S(x, \lambda) d t\right) \sin \left\{c_{2}+\int_{a}^{b} T(x, \lambda) d x\right\} T(b, \lambda) \\
= & b_{1}^{\prime} \lambda-b_{1} .
\end{aligned}
$$

From the last two equalities we have

$$
\begin{aligned}
& c_{1}=\frac{b_{2}-b_{2}^{\prime} \lambda}{\exp \left(\int_{a}^{b} S(x, \lambda) d x\right) \cos \left\{\tan ^{-1} F_{1}(b, \lambda)\right\}}, \\
& c_{2}=\tan ^{-1} F_{1}(b, \lambda)-\int_{a}^{b} T(x, \lambda) d x
\end{aligned}
$$

Substituting the values of $c_{1}$ and $c_{2}$ into (2.4) proves the result and,

(ii) if $b_{2}^{\prime}=0$, we obtain similarly from (2.4), (2.5) and (3.2)

$$
\begin{aligned}
\Phi(b, \lambda)= & c_{1} \exp \left(\int_{a}^{b} S(x, \lambda) d x\right) \cos \left\{c_{2}+\int_{a}^{b} T(x, \lambda) d x\right\}=b_{2}, \\
\Phi^{\prime}(b, \lambda)= & c_{1} S(b, \lambda) \exp \left(\int_{a}^{b} S(x, \lambda) d x\right) \cos \left\{c_{2}+\int_{a}^{b} T(x, \lambda) d x\right\} \\
& -c_{1} \exp \left(\int_{a}^{b} S(x, \lambda) d t\right) \sin \left\{c_{2}+\int_{a}^{b} T(x, \lambda) d x\right\} T(b, \lambda) \\
= & b_{1}^{\prime} \lambda-b_{1} .
\end{aligned}
$$

Hence from the last two equalities we evaluate

$$
\begin{aligned}
& c_{1}=\frac{b_{2}}{\exp \left(\int_{a}^{b} S(x, \lambda) d x\right) \cos \left\{\cot ^{-1} F_{2}(b, \lambda)\right\}} \\
& c_{2}=\cot ^{-1} F_{2}(b, \lambda)-\int_{a}^{b} T(x, \lambda) d x
\end{aligned}
$$

Substituting the values of $c_{1}$ and $c_{2}$ into (2.4) proves the theorem.

Theorem 2 Let $\Psi(t, \lambda)$ and $\Phi(t, \lambda)$ be the solutions of (1.1) satisfying (3.1) and (3.2), respectively. Then we find as $\lambda \rightarrow \infty$,

$$
\Psi(t, \lambda)=c \lambda^{3 / 2} \sin \lambda^{1 / 2}(t-a)-\frac{c}{2} \lambda\left(\int_{a}^{t} q(x) d x\right) \cos \lambda^{1 / 2}(t-a)+O(\lambda \eta(\lambda)),
$$

and 
(i) if $b_{2}^{\prime} \neq 0$,

$$
\begin{aligned}
\Phi(t, \lambda)= & -b_{2}^{\prime} \lambda \cos \lambda^{1 / 2}(b-t)-\lambda^{1 / 2}\left(b_{1}^{\prime}+\frac{b_{2}^{\prime}}{2} \int_{t}^{b} q(x) d x\right) \\
& \times \sin \lambda^{1 / 2}(b-t)+O\left(\lambda^{1 / 2} \eta(\lambda)\right),
\end{aligned}
$$

(ii) if $b_{2}^{\prime}=0$,

$$
\begin{aligned}
\Phi(t, \lambda)= & -b_{1}^{\prime} \lambda^{1 / 2} \sin \lambda^{1 / 2}(b-t)+\left(b_{2}+\frac{b_{1}^{\prime}}{2} \int_{t}^{b} q(x) d x\right) \\
& \times \cos \lambda^{1 / 2}(b-t)+O(\eta(\lambda)),
\end{aligned}
$$

where $\eta(\lambda)$ is as in (2.7).

Proof of Theorem 2 We evaluate the terms in Theorem 1 as $\lambda \rightarrow \infty$. Firstly, using (2.14) and (2.15) together with the series expansion, we find

$$
\begin{aligned}
\frac{T(a, \lambda)}{-c \lambda^{2}-d \lambda-e+S(a, \lambda)} \\
=\frac{\lambda^{1 / 2}-\cos \left(2 \lambda^{1 / 2} a+\zeta_{a}\right)+O\left(\eta^{2}(\lambda)\right)}{-c \lambda^{2}-d \lambda-e-\sin \left(2 \lambda^{1 / 2} a+\zeta_{a}\right)+O\left(\eta^{2}(\lambda)\right)} \\
=\frac{\lambda^{1 / 2}-\cos \left(2 \lambda^{1 / 2} a+\zeta_{a}\right)+O\left(\eta^{2}(\lambda)\right)}{-c \lambda^{2}\left[1+\frac{d}{c} \lambda^{-1}+\frac{e}{c} \lambda^{-2}+\frac{1}{c} \lambda^{-2} \sin \left(2 \lambda^{1 / 2} a+\zeta_{a}\right)+O\left(\lambda^{-2} \eta^{2}(\lambda)\right)\right]} \\
=\left[-\frac{1}{c} \lambda^{-3 / 2}+\frac{1}{c} \lambda^{-2} \cos \left(2 \lambda^{1 / 2} a+\zeta_{a}\right)+O\left(\lambda^{-2} \eta^{2}(\lambda)\right)\right] \\
\quad \times\left[1-\frac{d}{c} \lambda^{-1}-\frac{e}{c} \lambda^{-2}-\frac{1}{c} \lambda^{-2} \sin \left(2 \lambda^{1 / 2} a+\zeta_{a}\right)+O\left(\lambda^{-2} \eta^{2}(\lambda)\right)\right] \\
=-\frac{1}{c} \lambda^{-3 / 2}+O\left(\lambda^{-2} \eta(\lambda)\right) .
\end{aligned}
$$

From the last equality we have

$$
\cot ^{-1}\left[\frac{T(a, \lambda)}{-c \lambda^{2}-d \lambda-e+S(a, \lambda)}\right]=\frac{\pi}{2}+\frac{1}{c} \lambda^{-3 / 2}+O\left(\lambda^{-2} \eta(\lambda)\right),
$$

and from that

$$
\begin{aligned}
\cos \left\{\cot ^{-1}\left[\frac{T(a, \lambda)}{-c \lambda^{2}-d \lambda-e+S(a, \lambda)}\right]\right\} & =-\sin \left[\frac{1}{c} \lambda^{-3 / 2}+O\left(\lambda^{-2} \eta(\lambda)\right)\right] \\
& =-\frac{1}{c} \lambda^{-3 / 2}+O\left(\lambda^{-2} \eta(\lambda)\right), \\
\sin \left\{\cot ^{-1}\left[\frac{T(a, \lambda)}{-c \lambda^{2}-d \lambda-e+S(a, \lambda)}\right]\right\} & =\cos \left[\frac{1}{c} \lambda^{-3 / 2}+O\left(\lambda^{-2} \eta(\lambda)\right)\right] \\
& =1-\frac{1}{2 c^{2}} \lambda^{-3}+O\left(\lambda^{-7 / 2} \eta(\lambda)\right),
\end{aligned}
$$


and

$$
\begin{aligned}
\frac{1}{\cos \left\{\cot ^{-1}\left[\frac{T(a, \lambda)}{-c \lambda^{2}-d \lambda-e+S(a, \lambda)}\right]\right\}} & =\frac{1}{-\frac{1}{c} \lambda^{-3 / 2}+O\left(\lambda^{-2} \eta(\lambda)\right)} \\
& =-c \lambda^{3 / 2}+O(\lambda \eta(\lambda)) .
\end{aligned}
$$

Using $\int_{a}^{t} S(x, \lambda) d x$ given by $(2.17)$ we get

$$
\begin{aligned}
\exp \left(\int_{a}^{t} S(x, \lambda) d x\right)= & 1+\frac{1}{2 \lambda^{1 / 2}}\left\{\cos \left(2 \lambda^{1 / 2} t+\zeta_{t}\right)-\cos \left(2 \lambda^{1 / 2} a+\zeta_{a}\right)\right\} \\
& +O\left(\lambda^{-1 / 2} \eta^{2}(\lambda)\right)
\end{aligned}
$$

and also, using $\int_{a}^{t} T(x, \lambda) d x$ given by (2.18),

$$
\begin{aligned}
\sin \left(\int_{a}^{t} T(x, \lambda) d x\right)= & \sin \left[\lambda^{1 / 2}(t-a)-\frac{1}{2 \lambda^{1 / 2}} \int_{a}^{t} q(x) d x\right] \\
& +O\left(\lambda^{-1 / 2} \eta(\lambda)\right), \\
\cos \left(\int_{a}^{t} T(x, \lambda) d x\right)= & \cos \left[\lambda^{1 / 2}(t-a)-\frac{1}{2 \lambda^{1 / 2}} \int_{a}^{t} q(x) d x\right] \\
& +O\left(\lambda^{-1 / 2} \eta(\lambda)\right) .
\end{aligned}
$$

Hence

$$
\begin{aligned}
\cos \left\{\cot ^{-1}\left[\frac{T(a, \lambda)}{-c \lambda^{2}-d \lambda-e+S(a, \lambda)}+\int_{a}^{t} T(x, \lambda) d x\right]\right\} \\
=-\sin \left[\lambda^{1 / 2}(t-a)-\frac{1}{2 \lambda^{1 / 2}} \int_{a}^{t} q(x) d x\right]-\frac{1}{c} \lambda^{-1 / 2} \\
\quad \times \cos \left[\lambda^{1 / 2}(t-a)-\frac{1}{2 \lambda^{1 / 2}} \int_{a}^{t} q(x) d x\right]+O\left(\lambda^{-1 / 2} \eta(\lambda)\right) .
\end{aligned}
$$

Substituting the values of (3.19), (3.20) and (3.23) into $\Psi(t, \lambda)$ as in Theorem 1 and using trigonometric expansions, we obtain $\Psi(t, \lambda)$ as required. In order to find $\Phi(t, \lambda)$ as $\lambda \rightarrow \infty$ :

(i) if $b_{2}^{\prime} \neq 0$, using (2.14) and (2.15), we obtain

$$
\frac{S(b, \lambda)}{T(b, \lambda)}=\frac{O\left(\eta^{2}(\lambda)\right)}{\lambda^{1 / 2}\left[1+O\left(\lambda^{-1 / 2} \eta^{2}(\lambda)\right)\right]}=O\left(\lambda^{-1 / 2} \eta^{2}(\lambda)\right)
$$

and

$$
\begin{aligned}
\frac{b_{1}^{\prime} \lambda-b_{1}}{\left(b_{2}^{\prime} \lambda-b_{2}\right) T(b, \lambda)} & =\frac{b_{1}^{\prime} \lambda-b_{1}}{b_{2}^{\prime} \lambda^{3 / 2}-b_{2} \lambda^{1 / 2}+O\left(\lambda \eta^{2}(\lambda)\right)} \\
& =\frac{b_{1}^{\prime} \lambda-b_{1}}{b_{2}^{\prime} \lambda^{3 / 2}\left[1-\frac{b_{2}}{b_{2}^{\prime}} \lambda^{-1}+O\left(\lambda^{-1 / 2} \eta^{2}(\lambda)\right)\right]} \\
& =\left[\frac{b_{1}^{\prime}}{b_{2}^{\prime}} \lambda^{-1 / 2}-\frac{b_{1}}{b_{2}^{\prime}} \lambda^{-3 / 2}\right] \times\left[1+\frac{b_{2}}{b_{2}^{\prime}} \lambda^{-1}+O\left(\lambda^{-1 / 2} \eta^{2}(\lambda)\right)\right] \\
& =\frac{b_{1}^{\prime}}{b_{2}^{\prime}} \lambda^{-1 / 2}+O\left(\lambda^{-1} \eta^{2}(\lambda)\right) .
\end{aligned}
$$


Substituting the values of (3.24) and (3.25) into $F_{1}(b, \lambda)$ as in Theorem $\left.1(i)\right)$, we get

$$
F_{1}(b, \lambda)=\frac{b_{1}^{\prime}}{b_{2}^{\prime}} \lambda^{-1 / 2}+O\left(\lambda^{-1 / 2} \eta^{2}(\lambda)\right)
$$

From that

$$
\begin{aligned}
& \tan ^{-1} F_{1}(b, \lambda)=\frac{b_{1}^{\prime}}{b_{2}^{\prime}} \lambda^{-1 / 2}+O\left(\lambda^{-1 / 2} \eta^{2}(\lambda)\right) \\
& \cos \left\{\tan ^{-1} F_{1}(b, \lambda)\right\}=1-\frac{1}{2}\left(\frac{b_{1}^{\prime}}{b_{2}^{\prime}}\right)^{2} \lambda^{-1}+O\left(\lambda^{-1} \eta^{2}(\lambda)\right)
\end{aligned}
$$

and

$$
\sin \left\{\tan ^{-1} F_{1}(b, \lambda)\right\}=\frac{b_{1}^{\prime}}{b_{2}^{\prime}} \lambda^{-1 / 2}+O\left(\lambda^{-1 / 2} \eta^{2}(\lambda)\right) .
$$

From (3.27), we find

$$
\begin{aligned}
\frac{b_{2}-b_{2}^{\prime} \lambda}{\cos \left\{\tan ^{-1} F_{1}(b, \lambda)\right\}} & =\frac{b_{2}-b_{2}^{\prime} \lambda}{1-\frac{1}{2}\left(\frac{b_{1}^{\prime}}{b_{2}^{\prime}}\right)^{2} \lambda^{-1}+O\left(\lambda^{-1} \eta^{2}(\lambda)\right)} \\
& =\left(b_{2}-b_{2}^{\prime} \lambda\right) \times\left[1+\frac{1}{2}\left(\frac{b_{1}^{\prime}}{b_{2}^{\prime}}\right)^{2} \lambda^{-1}+O\left(\lambda^{-1} \eta^{2}(\lambda)\right)\right] \\
& =-b_{2}^{\prime} \lambda+\left[b_{2}-\frac{\left(b_{1}^{\prime}\right)^{2}}{2 b_{2}^{\prime}}\right]+O\left(\eta^{2}(\lambda)\right)
\end{aligned}
$$

Using $\int_{a}^{t} S(x, \lambda) d x$ given by (2.17) we get

$$
\exp \left(-\int_{t}^{b} S(x, \lambda) d x\right)=1+\frac{1}{2 \lambda^{1 / 2}} \cos \left(2 \lambda^{1 / 2} t+\zeta_{t}\right)+O\left(\lambda^{-1 / 2} \eta^{2}(\lambda)\right)
$$

and also, using $\int_{a}^{t} T(x, \lambda) d x$ given by (2.18)

$$
\begin{aligned}
\int_{t}^{b} T(x, \lambda) d x= & \int_{a}^{b} T(x, \lambda) d x-\int_{a}^{t} T(x, \lambda) d x \\
= & \lambda^{1 / 2}(b-t)+\frac{1}{2 \lambda^{1 / 2}}\left\{\sin \left(2 \lambda^{1 / 2} t+\zeta_{t}\right)-\int_{t}^{b} q(x) d x\right\} \\
& +O\left(\lambda^{-1 / 2} \eta^{2}(\lambda)\right) .
\end{aligned}
$$

From (3.27), (3.28) and (3.31) we see that

$$
\begin{aligned}
\cos \left\{\tan ^{-1} F_{1}(b, \lambda)-\int_{t}^{b} T(x, \lambda) d x\right\} \\
=\cos \left[\lambda^{1 / 2}(b-t)-\frac{1}{2 \lambda^{1 / 2}} \int_{t}^{b} q(x) d x\right] \\
+\frac{b_{1}^{\prime}}{b_{2}^{\prime}} \lambda^{-1 / 2} \sin \left[\lambda^{1 / 2}(b-t)-\frac{1}{2 \lambda^{1 / 2}} \int_{t}^{b} q(x) d x\right] \\
+O\left(\lambda^{-1 / 2} \eta(\lambda)\right) .
\end{aligned}
$$


Finally, substituting the values of (3.29), (3.30) and (3.32) into $\Phi(t, \lambda)$ as in Theorem 1(i)) and using trigonometric expansions, we complete the proof.

(ii) If $b_{2}^{\prime}=0$, the prove is similar to (i).

\section{Approximations for Green's function}

In this section, we obtain asymptotic approximations for Green's function of (1.1)-(1.3). Let $W_{t}(\Psi, \Phi)$ be the Wronskian of $\Psi(t, \lambda)$ and $\Phi(t, \lambda)$. We define $w(\lambda)$ as follows:

$$
w(\lambda):=W_{t}(\Psi, \Phi)=\Psi(t, \lambda) \Phi^{\prime}(t, \lambda)-\Psi^{\prime}(t, \lambda) \Phi(t, \lambda) .
$$

It is well known ([3]) that the Green's function of problem (1.1)-(1.3) is

$$
G(\varkappa, \xi, \lambda)= \begin{cases}\frac{\Psi(\varkappa, \lambda) \Phi(\xi, \lambda)}{w(\lambda)}, & a \leq \varkappa \leq \xi \leq b, \\ \frac{\Phi(\varkappa, \lambda) \Psi(\xi, \lambda)}{w(\lambda)}, & a \leq \xi \leq \varkappa \leq b .\end{cases}
$$

Theorem 3 For $a \leq \varkappa \leq \xi \leq b$, we find Green's function of problem (1.1)-(1.3) as $\lambda \rightarrow \infty$ :

(i) if $\circ_{2}^{\prime} \neq 0$,

$$
\begin{aligned}
G(\varkappa, \xi, \lambda)= & -\lambda^{-1 / 2} \frac{\sin \lambda^{1 / 2}(\varkappa-a) \cos \lambda^{1 / 2}(b-\xi)}{\cos \lambda^{1 / 2}(b-a)}+\frac{\lambda^{-1}}{\cos \lambda^{1 / 2}(b-a)} \\
& \times\left\{\frac{1}{2}\left(\int_{a}^{\varkappa} q(t) d t\right) \cos \lambda^{1 / 2}(\varkappa-a) \cos \lambda^{1 / 2}(b-\xi)\right. \\
& -\left[\frac{b_{1}^{\prime}}{b_{2}^{\prime}}+\frac{1}{2} \int_{\xi}^{b} q(t) d t\right] \sin \lambda^{1 / 2}(\varkappa-a) \sin \lambda^{1 / 2}(b-\xi) \\
& \left.+\frac{b_{1}^{\prime}}{b_{2}^{\prime}} \tan \lambda^{1 / 2}(b-a) \sin \lambda^{1 / 2}(\varkappa-a) \cos \lambda^{1 / 2}(b-\xi)\right\} \\
& +O\left(\lambda^{-1} \eta(\lambda)\right),
\end{aligned}
$$

(ii) if $b_{2}^{\prime}=0$,

$$
\begin{aligned}
G(\varkappa, \xi, \lambda)= & -\lambda^{-1 / 2} \frac{\sin \lambda^{1 / 2}(\varkappa-a) \sin \lambda^{1 / 2}(b-\xi)}{\sin \lambda^{1 / 2}(b-a)}+\frac{\lambda^{-1}}{\sin \lambda^{1 / 2}(b-a)} \\
& \times\left\{\frac{1}{2}\left(\int_{a}^{\varkappa} q(t) d t\right) \cos \lambda^{1 / 2}(\varkappa-a) \sin \lambda^{1 / 2}(b-\xi)\right. \\
& +\left[\frac{b_{2}}{b_{1}^{\prime}}+\frac{1}{2} \int_{\xi}^{b} q(t) d t\right] \sin \lambda^{1 / 2}(\varkappa-a) \cos \lambda^{1 / 2}(b-\xi) \\
& \left.-\frac{b_{2}}{b_{1}^{\prime}} \cot \lambda^{1 / 2}(b-a) \sin \lambda^{1 / 2}(\varkappa-a) \sin \lambda^{1 / 2}(b-\xi)\right\} \\
& +O\left(\lambda^{-1} \eta(\lambda)\right),
\end{aligned}
$$

where $\eta(\lambda)$ is as in (2.7). Similar results hold for $a \leq \xi \leq \varkappa \leq b$ exchanging the role of $\varkappa$ and $\xi$.

Proof of Theorem 3 (i) For the Wronskian, $w(\lambda)$, we need $\Psi^{\prime}(t, \lambda)$ and $\Phi^{\prime}(t, \lambda)$ which are obtained from $z^{\prime}(t, \lambda)$ given by (2.5). To obtain the derivation of $\Psi(t, \lambda)$, we substitute (3.6) 
and (3.7) into (2.5) and evaluate the terms as $\lambda \rightarrow \infty$. Hence

$$
\begin{aligned}
\Psi^{\prime}(t, \lambda)= & c \lambda^{2} \cos \lambda^{1 / 2}(t-a)+\frac{c}{2}\left(\int_{a}^{t} q(x) d x\right) \lambda^{3 / 2} \sin \lambda^{1 / 2}(t-a) \\
& +O\left(\lambda^{3 / 2} \eta(\lambda)\right) .
\end{aligned}
$$

Also substituting (3.10) and (3.11) into (2.5) and evaluating the terms as $\lambda \rightarrow \infty$, we have

$$
\begin{aligned}
\Phi^{\prime}(t, \lambda)= & -b_{2}^{\prime} \lambda^{3 / 2} \sin \lambda^{1 / 2}(b-t)+\left[b_{1}^{\prime}+\frac{b_{2}^{\prime}}{2} \int_{t}^{b} q(x) d x\right] \lambda \cos \lambda^{1 / 2}(b-t) \\
& +O(\lambda \eta(\lambda)) .
\end{aligned}
$$

Using $\Psi(t, \lambda), \Phi(t, \lambda)$ as in Theorem 2(i)) and (4.3), (4.4) in (4.1), we get

$$
\begin{aligned}
w(\lambda)= & c b_{2}^{\prime} \lambda^{3} \cos \lambda^{1 / 2}(b-a)+c b_{1}^{\prime} \lambda^{5 / 2} \sin \lambda^{1 / 2}(b-a) \\
& +O\left(\lambda^{5 / 2} \eta(\lambda)\right) .
\end{aligned}
$$

From this

$$
\begin{aligned}
\frac{1}{w(\lambda)}= & \frac{1}{\left\{c b_{2}^{\prime} \lambda^{3} \cos \lambda^{1 / 2}(b-a)\left[1+\frac{b_{1}^{\prime}}{b_{2}^{\prime}} \lambda^{-1 / 2} \tan \lambda^{1 / 2}(b-a)+O\left(\lambda^{-1 / 2} \eta(\lambda)\right)\right]\right\}} \\
= & \frac{1}{c b_{2}^{\prime} \cos \lambda^{1 / 2}(b-a)} \lambda^{-3}-\frac{b_{1}^{\prime}}{c\left(b_{2}^{\prime}\right)^{2}} \lambda^{-7 / 2} \frac{\sin \lambda^{1 / 2}(b-a)}{\cos ^{2} \lambda^{1 / 2}(b-a)} \\
& +O\left(\lambda^{-7 / 2} \eta(\lambda)\right) .
\end{aligned}
$$

Theorem 3(i) is proved by substituting $\Psi(t, \lambda), \Phi(t, \lambda)$ as in Theorem 2(i)) and (4.6) into (4.2). The other part (ii) can be proved similarly.

\section{Acknowledgements}

The authors would like to thank the referees for invaluable comments and insightful suggestions.

Funding

The current work is partially supported by Karadeniz Technical University.

\section{Competing interests}

The authors declare that they have no competing interests.

Authors' contributions

All authors read carefully and approved the final version of the manuscript.

\section{Publisher's Note}

Springer Nature remains neutral with regard to jurisdictional claims in published maps and institutional affiliations.

Received: 22 February 2017 Accepted: 2 May 2017 Published online: 16 May 2017

\section{References}

1. Levitan, BM, Sargsjan, IS: Sturm-Liouville and Dirac Operators. Kluwer Academic, Dordrecht (1991)

2. Annaby, MH, Tharwat, MM: On sampling theory and eigenvalue problems with an eigenparameter in the boundary conditions. SUT J. Math. 42(2), 157-176 (2006)

3. Fulton, CT: Two point boundary value problems with eigenvalue parameter contained in the boundary conditions. Proc. R. Soc. Edinb., Sect. A 77(3-4), 293-308 (1977)

4. Harris, BJ: The form of the spectral functions associated with Sturm-Liouville problems with continuous spectrum. Mathematika 44(1), 149-161 (1997) 
5. Coskun, H, Harris, BJ: Estimates for the periodic and semi-periodic eigenvalues of Hill's equation. Proc. R. Soc. Edinb., Sect. A 130, 991-998 (2000)

6. Coşkun, H, Başkaya, E: Asymptotics of the eigenvalues of regular Sturm-Liouville problems with eigenvalue parameter in the boundary condition for integrable potential. Math. Scand. 107(2), 209-223 (2010)

7. Coşkun, H, Kabataş, A: Asymptotic approximations of eigenfunctions for regular Sturm-Liouville problems with eigenvalue parameter in the boundary condition for integrable potential. Math. Scand. 113(1), 143-160 (2013)

Submit your manuscript to a SpringerOpen ${ }^{\circ}$ journal and benefit from:

- Convenient online submission

Rigorous peer review

- Immediate publication on acceptance

- Open access: articles freely available online

- High visibility within the field

- Retaining the copyright to your article 